\title{
Optimizing Resource Allocation in Hierarchically Distributed Data Centers
}

\author{
Rafael F. Vieira ${ }^{1}$, Carlos A. M. Teixeira ${ }^{1}$, Diego L. Cardoso ${ }^{1}$ \\ ${ }^{1}$ Laboratório de Pesquisa Operacional - Universidade Federal do Pará (UFPA) \\ Caixa Postal 479 - 66.075-110 - Belém - PA - Brazil \\ fogarollirafael@gmail.com, carlos.mattos@itec.ufpa.br, diego@ufpa.br
}

\begin{abstract}
The current network's infrastructure needs to support the rapidly increasing data traffic. Sophisticated planning approaches must be adopted by the operators so the high number of applications can be managed efficiently. In this work, a resource provisioning model for hierarchically distributed data centers is proposed using Integer Linear Programming (ILP). The objective is to increase the efficiency in the use of computational resources and decrease the overhead in network links. Results show that the model is able to efficiently accommodate $20 \%$ more applications when compared to the First-Fit approach.
\end{abstract}

\section{Introduction}

The growing volume of services and applications, in addition to the accelerated growth in wireless access demands, has posed significant challenges for the next generation of mobile networks. Cisco expects more than 50 billion individual devices to be connected to the network by 2020 [Computing 2015]. The traffic and processing capabilities required by these devices make it necessary to create more intelligent and sophisticated resource provisioning approaches than the currently used strategies.

Traditional cloud computing allocates the computational resources at the core of the network and is designed to be scalable. It is the current architecture of choice to host Internet services, as it is a data center (DC) based paradigm capable to manage and process large data volumes. However, traditional cloud computing incurs a significant delay in the response time of the applications and a traffic overhead in the network core, which is already at its limit, hindering stringent requirements such as latency of the applications.

The currently used architecture is not ready to support the stricter requirements of new services, e.g., virtual reality. In this way, new proposals are emerging to address these shortcomings such as Mobile Edge Computing (MEC), Cloudlet and Fog Computing. These proposals bring cloud computing (processing and storage) closer to the end user, thereby significantly reducing end-to-end latency, and enabling a multitude of new applications.

The MEC can be defined as an implementation of Edge Computing (EC) to bring computing capabilities to the edge within the radio access network, thus reducing latency and improving application contextualization [Dolui and Datta 2017]. MEC nodes or servers are usually located next to the radio network controller or a macro base station. However, the implementation of this new architecture presents several problems that need attention, such as the allocation and provisioning of resources, traffic control, higher capital expenditure (CAPEX) and operational expenditure (OPEX). 
Traditionally, the approaches proposed in the literature focus solely on a single layer of the architecture as in [da Silva et al. 2016], [Mei et al. 2017], [Upadhyaya and Ahuja 2017], ignoring the proliferation of DCs in the various layers of the network. They also overlook the DCs capacity to support a large amount of applications with different performance requirements. In this paper, a mathematical model was proposed with the objective of optimizing resources provisioning in hierarchically distributed networks.

This work considers a hierarchic network, divided into three levels, in which the MEC located on the edge coexists with the traditional cloud computing. The first level consists of the MEC architecture, the second level consists of a DC in a metropolitan network (MAN) and the third level is traditional cloud computing. The proposed model's objective is to provide a better resource provisioning so that the users can be served having their requirements respected, thus decreasing the use of network resources without affecting the quality of the service offered.

The remainder of this article is organized as follows: Section 2 discusses the related works, Section 3 presents the mathematical formulation of the proposed model, in Section 4 the results are discussed and Section 5 presents the conclusions obtained from this work.

\section{Related Work}

In recent years, the literature has been focusing on the deployment of DC resources to meet minimum Quality of Service (QoS) and Quality of Experience (QoE) requirements. A comprehensive model of MAN resource provisioning in a hybrid cloud computing and fog computing architecture is proposed in [Sturzinger et al. 2017], which decouples computing functions while accounting for traffic from a set of application profiles. The model proposed in [Sturzinger et al. 2017] intends to assist the MAN service provider in minimizing the total operational cost of IoT (Internet of Things) provisioning. The model demonstrates which application profile and topological parameters have the most significant effect on individual cost components.

A centralized and distributed resource allocation is presented in [Masoudi and Cavdar 2017], in which the algorithms defines the allocation of joint energy and channel distribution aiming at the minimization of energy consumption for mobile devices. The allocation algorithm proposed in [da Silva et al. 2016] is used to establish a strategy based on protection and in [Zhang et al. 2018] the authors present an optimal resource allocation scheme in heterogeneous networks with MEC. They formulate an optimization problem to provide an optimal computing unloading strategy, sub-channel allocation, uplink transmission power and resource scheduling. In [Zhang et al. 2017] the authors investigate the multiobjective resource allocation for multi-user MEC systems by adopting the utility of the system as the performance metric, which is a weighted combination of time and energy savings achieved by computing unloading.

The authors in [Chen et al. 2018] consider the edge cloud computing offloading which is composed of a set of wireless devices and each device has a renewable energy collection equipment. The authors formulate a multi-user multi-tasking computing download problem for the green MEC, and use the Lyanponuv optimization approach 
to determine the energy collection policy. In [Liao et al. 2017] the authors propose an intelligent MEC-based traffic accident detection system with proximity, low latency and vehicle identification, requiring the provision of computational resources for real-time responses.

The related works aims at meeting the requirements of the new applications through several techniques. However, these solutions consider aspects such as route optimization and resource maximization as well as do not consider the co-existence of DC at several network levels. Thus, this work aims at creating a model for a better provisioning and management of network resources.

\section{Mathematical Modeling}

The model proposes a strategy to allocate applications while optimizing the use of network resources in a hierarchically distributed scenario of three levels. The aim is to increase the use of resources and the number of served applications. The model consists of two main components: a set of $R$ applications and a set of $C$ nodes, representing the network's topology. This nodes are interconnected by fiber links. $R$ is the input of the model and the output consists of two variables: $X_{i, j}^{r}$, which represents the path an application of the set $R$ will take to reach it's destiny and $P_{r, i}$, the node chosen by each application for processing.

\subsection{Topology and Variables}

The proposed topology, composed of three levels of DCs, is illustrated in Figure 1. The set of nodes $C$ that composes the topology is sub-divided into three types according to its positioning in the network, as follows: edge nodes, metropolitan data centers (MDCs) and core nodes or core data centers (CDCs). The computational capacity is a factor that decreases with the distance of the DC from the network core, thus the edge DCs have the lowest computational capacity.

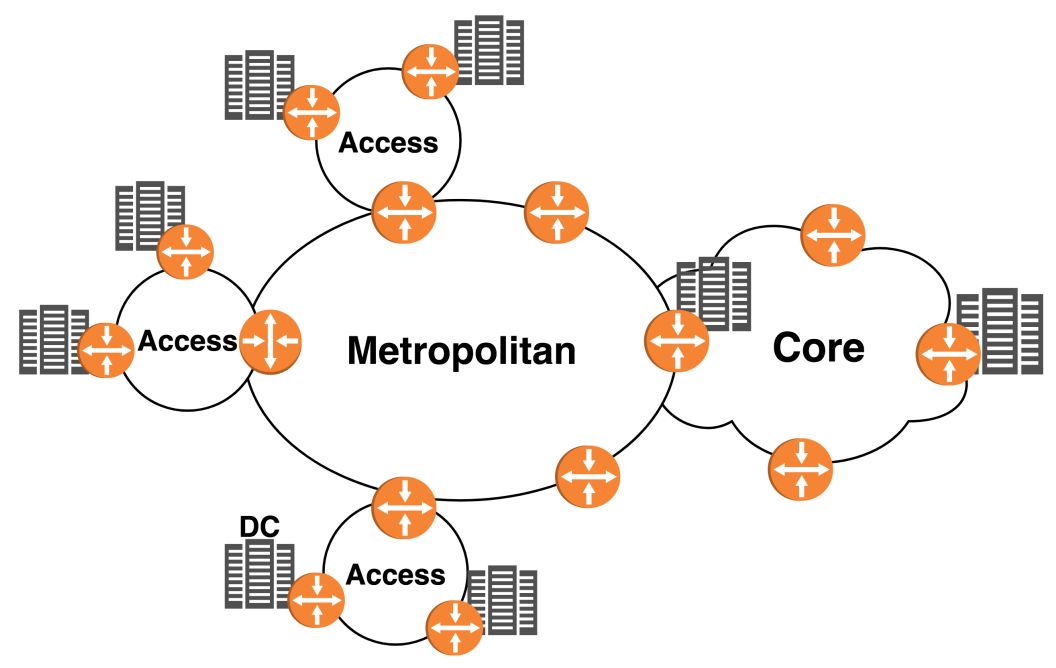

Figure 1. Three levels hierarchically distributed network topology.

The $C$ set is sub-divided into another sub-group composed of two types: routing nodes and processing nodes. Routing nodes are used to route de applications through the 
topology until a DC that is capable of processing them is found. Processing nodes can be DCs, the final destination of the application, or serve as routing nodes.

The set $C$ is defined by six parameters, as described below:

- $D C_{n}=1$ if node $n$ is a DC.

- $C P_{n}$ is the number of processing units (UP) in node $n$ has.

- $T_{i, j}=1$ if node $i$ is connected to node $j$.

- Dist $_{i, j}$ is the distance from the link that connects nodes $i$ and $j$.

- $W l_{i, j}$ is the total of wavelength in the fiber that links nodes $i$ and $j$.

- $V f$ is the data propagation speed in the fiber.

The set of applications $R$ consists of five elements, defined as follows:

- $I D_{r}$ represents the application id $r$.

- $S R C_{r}$ represents the source of the application $r$.

- $P_{r}$ represents how many PUs are required to process an application $r$.

- $W_{r}$ represents how many wavelenghts the application $r$ will occupy in the path.

- $D_{r}$ represents the maximum allowed delay of the application $r$.

The proposed model uses two binary variables to represent the flow and allocation of the applications.

- $X_{i, j}^{r}=1$ if the path for application $r$ traverses link that goes from the node $i$ to the node $j$, it represents the flow of the application to its destination.

- $A r, i=1$ if the application $r$ is allocated in the DC node $i$.

\subsection{Objective Function}

The problem was modeled through a multiobjective function, which aims at maximizing the number of allocated applications while minimizing the number of resources used. The function is then defined to minimize the three components shown in Eq. (1):

$$
\text { MinimizeFO }=A l o c_{A}+\text { Aloc }_{P}+\text { Path }
$$

The first component in Eq. (1) refers to the allocation of the applications, the second component is the allocation of the PUs and the third component is the usage of fiber resources.

$$
A l o c_{A}=T_{A}-\sum_{r \in R} \sum_{n \in C} A_{r, n}
$$

Eq. (2) represents the amount of applications that could not be allocated. Where TA is the total of applications that exist at an instant of time and the sum represents the total of applications that have been allocated. So, minimizing Eq. (2) maximizes the number of allocated applications. 


$$
\operatorname{Aloc}_{P}=T_{P}-\sum_{r \in R} \sum_{n \in C} A_{r, n} * P_{r}
$$

Eq. (3) represents the amount of PUs that were not used. Where $T_{P}$ is the total of PUs in the network and the sum represents the total number of UPs used by the solution. Thus, when Eq. (3) is minimized, the use of computational resources is maximized.

$$
\text { Path }=\sum_{r \in R} \sum_{i \in C} \sum_{j \in C} X_{i, j}^{r}
$$

Eq. (4) computes the total of fiber resources used by the solution. The sum represents the total number of hops made by the applications before reaching the final destination, i.e., the DC. So, by minimizing the number of hops in an application the usage of fiber resources can be minimized.

\subsection{Constraints}

A set of constraints is necessary to ensure that network and application constraints are respected, such as the minimum acceptable latency of an application or even if the maximum amount of PU that a DC can support is not exceeded, that is ensured by Eq. (5), (6) and (7), respectively.

$$
\begin{gathered}
\sum_{r \in R}\left(A_{r, n} * P_{r}\right) \leq C P_{n}, \forall n \in C \\
\sum_{i \in C} \sum_{j \in C} \frac{X_{i, j}^{r} * D_{i s t_{i, j}}}{V f} \leq D_{r}, \forall r \in R \\
\sum_{r \in R} X_{i, j}^{r} * W_{r} \leq W l_{i, j}, \forall(i \in C, j \in C)
\end{gathered}
$$

Eq. (5) ensures that a DC will not exceed its capacity, that is, at most $C P_{n}$ UPs will be allocated on that node. Nodes only have routing capabilities, i.g., $C P n=0$, so it is not possible to allocate applications in them. Eq. (6) ensures that an application will have its maximum allowed latency. Eq. (7) constrains the fiber capacity by limiting the total of applications that use the fiber. Applications that are unable to use the fiber are blocked. Eq. (8)-(15) guarantee the path of the application, making it travel valid paths.

$$
\begin{gathered}
\sum_{k i n C} X_{n, k}^{r}+P_{r, n}=1, \forall\left(r \in R, n \in C, S R C_{r}=n, D C_{n}=1, T_{n, k}=1\right) \\
\sum_{k \in C} X_{n, k}^{r}=1, \forall\left(r \in R, n \in C, S R C_{r}=n, D C_{n}=0, T_{n, k}=1\right)
\end{gathered}
$$

Eq. (8) and (9) treats the application on its source node. Eq. (8) ensures that the application can be allocated on the current node or move to a next node. Eq. (9) ensures that the application should proceed to a next node, seeking a DC capable of attending it. 


$$
\begin{gathered}
\sum_{k \in C} X_{n, k}^{r} \leq 1, \forall\left(r \in R, n \in C, S R C_{r} \neq n, D C_{n}=1\right) \\
\sum_{k \in C} X_{n, k}^{r}-\sum_{i \in C} X_{i, n}^{r}=0, \forall\left(r \in R, n \in C, S R C_{r} \neq n, D C_{n}=0\right)
\end{gathered}
$$

Eq. (10) guarantee that if the $r$ application is on a node that is a DC and it can be allocated in it or continue searching for a processing node. Eq. (11) ensures that if the $r$ application is on a routing node it continues its search for a DC.

$$
\begin{gathered}
\sum_{k \in C} X_{j, k}^{r}=0, \forall\left(T_{j, k}=0, r \in R\right) \\
X_{j, k}^{r}+X_{k, j}^{r} \leq 1, \forall(r \in R, j \in C, k \in C, j \neq k)
\end{gathered}
$$

The Eq. (12) ensures that the application $\mathrm{r}$ goes from node $j$ to node $k$ only if there is a link connecting these two nodes, preventing an application from going from one node to a non-interconnected node. The Eq. (13) ensures that an application will not loop in between two nodes, that is, if an application $r$ went from node $i$ to node $j$ it can not go back through the same link

$$
\begin{gathered}
\sum_{n \in C} A_{r, n} \leq 1, \forall r \in R \\
A_{r, j}-\sum_{k \in C} X_{k, j}^{r} \leq 0, \forall\left(r \in R, j \in C, D C_{j}=1\right)
\end{gathered}
$$

Eq. (14) ensures that the application $r$ will be allocated in at most one DC. Eq. (15) ensures that the application will be allocated to the end node of your course. Thus, node $j$ is the last node in the application path $r$.

\section{Results}

The mathematical model was implemented using the IBM ILOG CPLEX Optimization Studio optimization software. The scenario parameters are presented below:

- 258 Applications, summing up to $2600 \mathrm{PU}$.

- $1500 \mathrm{PU}$ at the network core.

- 500 PU on the metro network.

- $600 \mathrm{PU}$ at the edge of the network.

- 6 DCs (4 on the edge, 1 on the metro and 1 on the core).

- 19 Links interconnecting the nodes. 
The applications used as input to the model (in both approaches) were generated using a linear probability distribution, until the sum of the processing requirements of the applications is equal to 2600 PU. As a benchmark, an approach was implemented based on First-Fit [10], available in the literature. In the First-Fit scheme, the applications searching over the DCs, from edge to core, until the DC with available capacity, is found. In both approaches the applications search for the shortest routing path (least number of hops) to the DC in which the application will be allocated.

A set of seven applications, as pointed out in [Sturzinger et al. 2017], is represented in table 1 along with their delay and processing parameters. It is considered as delay the time required by each application to reach the DC.

Table 1. Application Profiles [Sturzinger et al. 2017]

\begin{tabular}{|c|c|c|}
\hline Application & Delay (ms) & PU (CPU/Mbps) \\
\hline Virtual Reality & 10 & 30 \\
\hline Industrial Automation & 20 & 9 \\
\hline Data Backup & 1000 & 0 \\
\hline Smart Grid & 50 & 7 \\
\hline Smart Home & 60 & 0 \\
\hline Health & 40 & 20 \\
\hline Tactile Internet & 1 & 5 \\
\hline
\end{tabular}

For evaluation purposes, 3 different scenarios have been created, as following: Scenario 1 - Hierarchy of data centers (DCs in the 3 levels, as proposed); Scenario 2 Existence of DCs in the core only; Scenario 3 - DCs only at the edge. As can be observed in the results in Fig. 2 and Fig. 3, the proposed model achieved superior results both in the allocation of the applications and in DC utilization. The improvement obtained in the proposed technique is due to a better distribution of the applications in the scenario, avoiding congestion and early use of DCs.

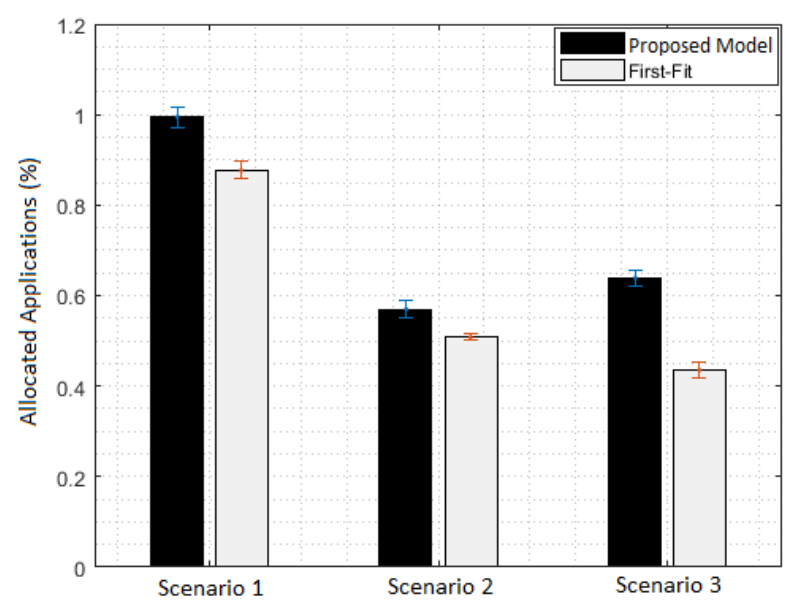

Figure 2. Allocated Applications

It is possible to identify that the proposed model was superior to the benchmark in 
the use of data centers and the number of applications that have been allocated, as shown in Fig. 2 and 3. Fig. 2 shows an increase of approximately $40 \%$ of allocated applications between scenarios 1 and 3 for the proposed model. In scenario 1 there is a difference of more than $10 \%$ from the proposed model to the benchmark. The obtained results show that, with better network resources provisioning it is possible to allocate a larger number of applications.

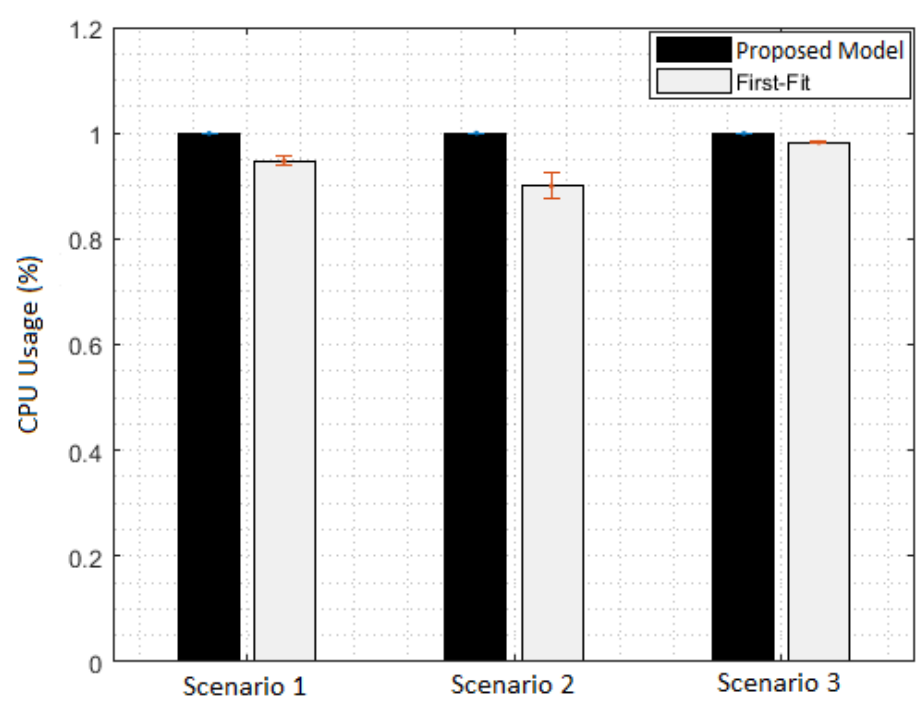

Figure 3. Processing Units Usage

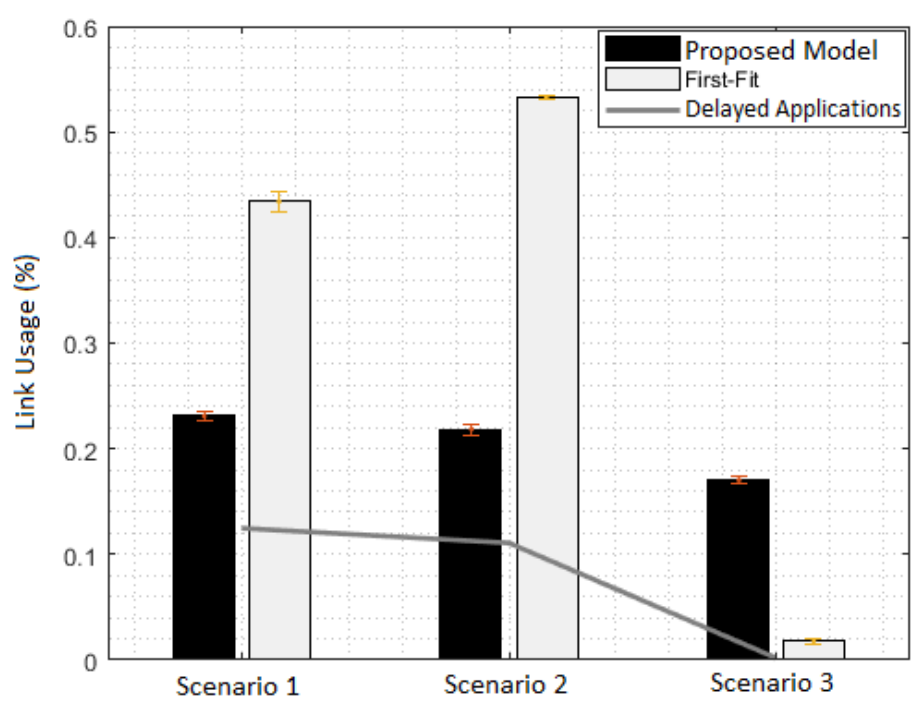

Figure 4. Link Usage

In Fig. 4 the usage of fiber resources for each approach is presented. It can be observed that First-Fit used much more fiber resources than the proposed model, except in scenario 3. The usage of fiber in scenario 3 is justified since the proposed model looks 
for the best way to increase the number of allocated applications while in the benchmark there is no such concern. In the First-Fit scheme, the allocation to other DCs is not necessary if there is available capacity on the source node. Thus, the number of allocated applications and use of fibers are decreased.

Fig. 4. also shows the percentage of applications that were not allocated in the benchmark, due to delay restrictions. In the proposed model the applications do not have significant delays.

The reason for these applications not being allocated is the non-optimized usage of the fiber resources, overloading the links of smaller paths, consequently obligating the demands to get a not optimized route, thus not meeting the QoS requirements.

\section{Conclusion}

Data Center architectures has emerged as a facilitator in applications processing for lowcapacity hardware. However, due to new performance requirements, the traditional cloud computing model has become insufficient. In this work, a mathematical model was developed to optimize application allocation in hierarchical DC networks. The proposed model was able to allocate more applications by making better use of the network resources than the benchmark approach, which is based in the first DC found.

According to the obtained results, there was a $20 \%$ improvement in the application allocation capacity, in addition to a $30 \%$ reduction in fiber usage when compared to First-Fit. Also, the QoS requirements for different applications of the next generation were fulfilled. In future work, a heuristic will be developed, to optimize the allocation of network resources in order to meet the needs of all users more efficiently, looking for sub-optimal but faster answers.

\section{References}

Chen, W., Wang, D., and Li, K. (2018). Multi-user multi-task computation offloading in green mobile edge cloud computing. IEEE Transactions on Services Computing.

Computing, F. (2015). the internet of things: Extend the cloud to where the things are. Available on: http://www. cisco. com/c/dam/en_us/solutions/trends/iot/docs/computingoverview. pdf.

da Silva, C. N., Wosinska, L., Spadaro, S., Costa, J. C., Francês, C. R., and Monti, P. (2016). Restoration in optical cloud networks with relocation and services differentiation. Journal of Optical Communications and Networking, 8(2):100-111.

Dolui, K. and Datta, S. K. (2017). Comparison of edge computing implementations: Fog computing, cloudlet and mobile edge computing. In Global Internet of Things Summit (GIoTS), 2017, pages 1-6. IEEE.

Liao, C., Shou, G., Liu, Y., Hu, Y., and Guo, Z. (2017). Intelligent traffic accident detection system based on mobile edge computing. In Computer and Communications (ICCC), 2017 3rd IEEE International Conference on, pages 2110-2115. IEEE.

Masoudi, M. and Cavdar, C. (2017). Cloud vs edge computing for mobile services: Delay-aware decision making to minimize energy consumption. arXiv preprint arXiv:1711.03771. 
Mei, J., Li, K., and Li, K. (2017). Customer-satisfaction-aware optimal multiserver configuration for profit maximization in cloud computing. T-SUSC, 2(1):17-29.

Sturzinger, E., Tornatore, M., and Mukherjee, B. (2017). Application-aware resource provisioning in a heterogeneous internet of things. In Optical Network Design and Modeling (ONDM), 2017 International Conference on, pages 1-6. IEEE.

Upadhyaya, J. and Ahuja, N. J. (2017). Quality of service in cloud computing in higher education: A critical survey and innovative model. In I-SMAC (IoT in Social, Mobile, Analytics and Cloud)(I-SMAC), 2017 International Conference on, pages 137-140. IEEE.

Zhang, J., Xia, W., Yan, F., and Shen, L. (2018). Joint computation offloading and resource allocation optimization in heterogeneous networks with mobile edge computing. IEEE Access, 6:19324-19337.

Zhang, X., Mao, Y., Zhang, J., and Letaief, K. B. (2017). Multi-objective resource allocation for mobile edge computing systems. In Personal, Indoor, and Mobile Radio Communications (PIMRC), 2017 IEEE 28th Annual International Symposium on, pages 1-5. IEEE. 